\title{
Time is money. Waiting costs explain why selection favors steeper time discounting in deprived environments
}

\author{
Hugo Mell $^{1}$, Nicolas Baumard ${ }^{1}$, and Jean-Baptiste André ${ }^{1}$ \\ ${ }^{1}$ Institut Jean Nicod, Département d'études cognitives, ENS, EHESS, PSL \\ Research University, CNRS, Paris France
}

December 4, 2019

\begin{abstract}
Individuals exposed to deprivation tend to show a characteristic behavioural syndrome suggestive of a short time horizon. This pattern has traditionally been attributed to the intrinsically higher unpredictability of deprived environments, which renders waiting for long term rewards more risky (i.e. collection risks are high). In the current paper, based on a simple dynamic life history model, we show that a significant portion of individuals' propensity to discount future rewards might have a completely distinct origin. Upon collecting a resource, individuals have the opportunity to accumulate "capital" (e.g. grow muscular tissue, build a protective shelter, buy a car, etc.), which eventually increases their productivity and/or their chances of survival. As a result, delaying the collection of a resource creates an opportunity cost in the sense that during the waiting time, the benefits otherwise generated by the increment in capital are lost. These forgone benefits are independent of collection risks and constitute waiting costs per se. Using optimal control theory we show that these costs can lead to the evolution of short time horizons even in the complete absence of collection risks. Moreover, assuming diminishing returns to capital, we show that the evolutionarily stable time horizon increases with the amount of capital already owned by individuals. When individuals possess little capital, they have a lot of room to improve their productivity and/or survival, hence they should be impatient to collect resources; that is, their time horizon should be short. On the contrary, when individuals already possess a lot of capital, the benefits of further accumulation are plateauing, hence patience becomes a more profitable strategy and individuals should lengthen their time horizon. This means that individuals get more patient as they age and that people in deprivation, who still have important productive and survival needs that can be satisfied, should have a shorter time horizon. Moreover, beyond time horizon, our model shows that people with little capital should also be more risk averse than the more privileged. Taken together, these results lead us to interpret the behavioral constellation of deprivation in a new way.
\end{abstract}




\section{Introduction}

People living in conditions of deprivation tend to exhibit a constellation of correlated behaviours across a variety of domains (Mell et al., 2017b; Pepper and Nettle, 2017). For example, people of lower socioeconomic status tend to disinvest in their future health (Pepper and Nettle, 2014), show higher rates of teen pregnancy (Dickins et al., 2012) and are more likely to drop out of school (Winding and Andersen, 2015). This cluster of behaviours is thought to arise at least partly from individual differences in time discounting, that is, variation in people's propensity to discount future benefits and costs. Empirical measures of discount rates across individuals and societies indeed show a significant amount of variation, which correlates with indicators of deprivation (e.g. Bruderer Enzler et al., 2014; Green et al., 1996; Harrison et al., 2002; Tanaka et al., 2010; Wang et al., 2016), and correlations between people's tendency to discount the future and reproductive behaviours have also been documented (Bulley and Pepper, 2017). Hence, a theoretical understanding of why environments should elicit different rates of delay discounting is needed.

The classical explanation states that a short-term orientation should be favored by natural selection in ecologies where the probability to get a reward at the end of a delay is low (i.e. the future is uncertain). Borrowing the terminology used in behavioural ecology (Fawcett et al., 2012; Houston et al., 1982; Stevens and Stephens, 2010), the last statement is equivalent to say that delay discounting should increase when the collection risk associated to the delay is high. This therefore implies that we should be able to identify specific factors that increase collection risk in deprived ecologies.

Extrinsic mortality is the source of collection risk most frequently mentioned to explain differences in time horizon in human populations (Ellis et al., 2009; Nettle et al., 2012; Pepper and Nettle, 2014). Its theoretical relevance to explain variation in delay discounting is undoubted, as it captures the simplest form of collection risk. Indeed, the risk of dying or, more generally, of being incapacitated during the waiting period is one reason why a reward might never be collected. Moreover, sources of extrinsic mortality generally increase with deprivation, which can sometimes lead to substantial disparities between the most deprived and most affluent areas (Shaw et al., 2005). Nevertheless, several traits of the so-called behavioural constellation of deprivation involve decisions about costs or rewards that are delayed over relatively short timescales (e.g. days, months, a few years). In such contexts, variation in extrinsic mortality rates will not usually be large enough to explain differences in people's time horizon (Mell et al., 2017a; Riis-Vestergaard and Haushofer, 2017). For example, as pointed out by Riis-Vestergaard and Haushofer (2017), in a study reporting data on temporal discounting from 53 different countries (Wang et al., 2016), people discount $46 \%$ over one year while the average mortality risk per year in the countries in the dataset is only $0.148 \%$, which can only account for $0.13 \%$ of the actual discounting. Other factors must therefore be considered to account for observed discount rates.

Additional sources of collection risk beyond extrinsic mortality could explain part of the increase in delay discounting in deprived environments (e.g. high competition or less trustworthy social partners that lead to greater volatility). In this paper, however, we aim to highlight that time horizons can also vary for reasons that have nothing to do with collection risk. We use optimal control theory (Pontriagin et al., 1986) to analyse a 
simple dynamic life history model, where individuals can use the resources they gain at every instant of their life either to reproduce or to increase their personal capital (where by capital we mean both the individual's biological organism, that is the embodied capital in the sense of Kaplan et al., 2000, and more generally all the assets that determine an individual's ability to exploit the environment). Several attempts to formalize the evolution of time preferences have been made in the economic litterature in order to understand the origins and derive the optimal age-specific trajectory of individuals' discount rates (Chu et al., 2010; Hansson and Stuart, 1990; Kageyama, 2009; Rogers, 1994; Sozou and Seymour, 2003). In particular, Chu et al. (2010) analysed a life history model with endogenous body size and energy production. They show that individuals' time horizon does not depend only on age-specific mortality rates once the process of ressource allocation during growth is taken into account. Here, we report similar results and explore how they relate to differences in discounting across levels of deprivation.

First, as Chu et al. (2010), we show that it can be adaptive for individuals to discount the future independently of the existence or not of collection risks. Even if it is eventually collected with absolute certainty, a resource obtained after a delay often has an intrinsically lower value than the same resource obtained immediately. This is because, upon collecting a resource, an individual can invest that resource into her personal capital (e.g., the maintenance of her biological body, the construction of a shelter, etc.), and this supplementary capital eventually allows her to be more productive (that is, to produce more resources in the future than what she would have produced otherwise) and/or to be better protected against hazards (that is, to reach a lower mortality rate or, more generally, a lower rate of degradation of her capital). When an individual chooses a delayed resource over an immediate one, she thus renounces all the benefits this supplementary capital would have generated during that delay. These opportunity costs are independent of collection risks and constitute waiting costs per se.

Second, under the hypothesis that capital has diminishing returns (Charnov, 1993), we show that the actual value of waiting costs for an individual at any given time of her life decreases with the amount of capital she already possesses at that time. An individual's waiting costs are equal to the marginal benefit of the supplementary capital she renounces to while waiting. The lower this marginal benefit is, the less costly it is to wait. As a result, if capital has diminishing returns (that is, if the marginal benefit of each additional unit of capital decreases as individuals grow), individuals who have already accumulated a lot of personal capital should be relatively patient because each supplementary unit of capital makes a small difference to them, whereas those who have accumulated little should have a short time horizon as each additional resource can make a big difference to them.

Third, from this model we seek to understand the effect of deprivation on time horizon. On the one hand, we show that individuals should discount the future less steeply if they live in an environment where productivity and survival cannot be substantially improved by investing into capital, because their return on capital is always lower and therefore the waiting costs are also lower. On the other hand, we show that individuals should be more short-term oriented if the basic capital they own from birth is low, either because they received little support from their parents or because their society as a whole provides little social care. Together with the empirical observations that people living in poverty tend to discount the future more, these results lead us to suggest that, in most cases, 
deprivation does not consist in living in an environment that can hardly be improved, but rather in possessing little at birth and having to build everything from scratch.

The finding that the evolutionarily stable discounting increases with the magnitude of returns to growth mirrors, at the level of individual growth, the classic result in life history theory that earlier reproductive effort is favoured in growing populations compared to populations at demographic equilibrium (Abrams, 1993; Caswell, 2007; Dańko et al., 2017). In a growing population, reproducing early is favoured because offsprings produced early themselves contribute quickly to the expansion of the lineage. Similarly, at the level of the individual, units of capital yield a greater overall contribution and are therefore more urgent to obtain when there is room for growth.

To picture more intuitively how waiting costs can steepen discounting, one can think of someone living on the streets, facing a whole range of adverse conditions such as exposure to climatic hazards, severe limitations to participate in economic activities, risk of sleeep deprivation, and so on. For that person, the time spent without the amount of resource necessary to afford some form of accomodation is extremely costly. Any opportunity to rapidly improve his current state, even marginally, will appear very valuable over options that involve long delays. In the TV series Atlanta, the life of the main character Earnest Marks provides vivid illustrations of theses high waiting costs encountered by people living in poverty. In one particular episode, Earnest tries to trade his phone for cash in a pawn shop and is offered $90 \$$ for it. However, one of his friend convinces him to let him conduct instead a succession of trades that lead to a dog-breeding investment, expected to yield $800 \$$ eight months later. The reaction of Earnest as he learns that he will not get the money before several months illustrates perfectly how waiting costs can lead to steep discount rates:

Van needed that money... My daughter needed that money... Not in September, but today. See, I am poor Darius, and poor people don't have time for investments, because poor people are too busy trying not to be poor! I need to eat today, not in September...

\section{Model}

Here, we first model the evolution of the individuals' life history strategy. To do so, we use optimal control theory, and our model is essentially identical to Perrin et al. (1993) and Koziowski and Weiner (1997). In a second step, we show how the evolutionarily stable growth strategy determines the time horizon of individuals, and the level of risk they are willing to take.

\subsection{Evolutionarily stable growth schedule}

Individuals are characterized, at any age $t$, by their total amount of capital that we call here for simplicity their body weight $w(t)$. Body weight affects two parameters of individuals' life history via trade-offs. On the one hand, the body weight affects mortality, through a monotonous decreasing function $m(\cdot)$. On the other hand, body weight affects the amount of resources that the individual produces per unit of time, through a monotonically increasing productivity function $p(\cdot)$. 
Throughout this paper we assume that capital has diminishing returns both in productivity and in survival (that is, $\forall w>0, p^{\prime \prime}(w)<0$ and $m^{\prime \prime}(w)>0$ ). This assumption is standard in evolutionary biology (Charnov, 1993). From an evolutionary point of view, it is justified by the fact that natural selection should have shaped individuals' life history decisions in such a way that they invest in different components of their capital (different organs, or different non-biological assets) in decreasing order of priority from the most profitable down to the least profitable ones (Iwasa and Roughgarden, 1984; Perrin, 1992). As a result, the more advanced an individual is in his pyramid of needs, i.e. the more capital she has already accumulated, and the less each additional unit of capital makes a difference.

An individual's strategy consists in choosing, at each instant of her life, how she allocates the resources she produces at that moment. She can invest these resources in two activities: reproduction or growth. Formally, an individual's strategy is therefore an allocation function $u(\cdot) \in[0,1]$ that indicates the fraction of her resources that she allocates to growth at each age, the rest $(1-u(\cdot))$ being allocated to reproduction. It is therefore on this function that natural selection acts. The objective of the present approach is to characterize the nature of this function at an evolutionary equilibrium.

To do this, we consider a population subject to density-dependent regulation via fertility, in which one can show that natural selection maximizes the "lifetime reproductive success" of individuals (see Supporting Information Section 3 and see also e.g. André and Rousset, 2019; Dańko et al., 2017). In other words, selection favours the allocation function $u(\cdot)$ that maximizes the total amount of "reproductive investments" made by the individual over her lifetime, referred to here as $V_{0}$ (see SI Section 3 ) ; we note this quantity $V_{0}$ because it corresponds to an individual's reproductive value at birth (Fisher, 1930; Perrin and Sibly, 1993). What is more, under the same model of density-dependent regulation, when the environment is variable, one can show that natural selection favors the evolution of a reaction norm by which individuals maximize their life time reproductive success in each environment taken in isolation (see SI Section 3).

Let us consider an individual of age $t$. This individual is characterized by two variables: his capital, that is his body weight, $w(t)$, and his probability of still being alive, $l(t)$. The following system of differential equations gives us the dynamics of these two variables along the individual's life:

$$
\begin{aligned}
& \frac{d w(t)}{d t}=u(t) p[w(t)] \\
& \frac{d l(t)}{d t}=-m[w(t)] l(t)
\end{aligned}
$$

A function called the Hamiltonian is then defined:

$$
H(t) \equiv u(t) p[w(t)] \lambda(t)-l(t) m[w(t)] V(t)+(1-u(t)) p[w(t)] l(t)
$$

An intuitive way to understand the meaning of this function is to see it as the "marginal value" of the instant $t$. In other words, $H(t) d t$ represents how much the infinitesimal duration $d t$ has contributed to $V_{0}$. At each instant $t$ of her life, three things happen to the individual:

1. She invests a quantity of resources $u(t) p[w(t)]$ in the growth of her capital $w$. 
2. If she is still alive, she invests an amount of resources $(1-u(t)) p[w(t)]$ in reproduction.

3. Her probability of still being alive decreases by an amount $l(t) m[w(t)]$.

The objective of equation 3 is to measure the impact of these three events on the individual's total reproductive output $V_{0}$. To do this, these three changes are weighted by a measure of their respective "value" in terms of reproduction.

1. The investment into body weight is weighted by $\lambda(t)$ which represents the marginal value of body weight at time $t$. In other words, $\lambda(t)$ tells us how much it increases $V_{0}$ if we increase a little bit the individual's body weight at time $t$.

2. The investment in survival is weighted by $V(t)$ which represents the reproductive value of an individual of age $t$. $V(t)$ tells us how much $V_{0}$ increases if we increase a little bit the individual's probability of being alive at time $t$.

3. Investment in reproduction is weighted by $l(t)$ which represents the probability that the individual is still alive at age $t$, and therefore that the reproductive investment actually takes place.

The maximum principle (Pontriagin et al., 1986) states that the optimal allocation function consists in always investing, at each instant, so as to maximize the marginal value of instant $t, H(t)$. From equation equation 3 , the evolutionary stable allocation function $u(\cdot)$ must therefore obey (i) $u(t)=1$ iff $\lambda(t)>l(t)$, and (ii) $u(t)=0$ iff $l(t)>\lambda(t)$. In words, when the marginal value of one's body weight is larger than one's probability of still being alive, one must invest everything in growth, otherwise one must invest everything in reproduction.

To determine the evolutionarily stable allocation function we must therefore calculate the two functions $\lambda(t)$ and $l(t)$. For this purpose, we use the canonical equations of optimal control:

$$
\begin{aligned}
& \frac{d \lambda(t)}{d t}=-\frac{\partial H(t)}{\partial w(t)} \\
& \frac{d V(t)}{d t}=-\frac{\partial H(t)}{\partial l(t)}
\end{aligned}
$$

In short, the canonical equation tells us that the marginal value of $w$ and $l$ decreases over time by a value that corresponds to their instantaneous marginal value. This can be understood by considering the fact that the marginal value of a given state at time $t$ is equal to its instantaneous value in $t$ plus its later total marginal value (to gain a better intuitive understanding of these equations, we recommend Iwasa and Roughgarden, 1984).

In this model, the optimal strategy is "bang bang" (see Koziowski and Weiner, 1997; León, 1976; Perrin and Sibly, 1993; Taylor et al., 1974). The individual starts by investing all her resources in growth, until she reaches a switching age $t_{a}$, which is found as the solution of $\lambda\left(t_{a}\right)=l\left(t_{a}\right)$, then the individual must invest all her resources exclusively in reproduction, and maintain her body weight constant at its adult size $w\left(t_{a}\right)$. Our aim, now, is to calculate the evolutionarily stable age at maturity $t_{a}$ and adult size $w\left(t_{a}\right)$. To do so, we start by considering the pure reproduction phase. 


\subsubsection{Pure reproduction phase and derivation of the switching time}

For simplicity, we call $w_{a} \equiv w\left(t_{a}\right)$ the adult body weight, $m_{a} \equiv m\left[w_{a}\right]$ the adult mortality, and $p_{a} \equiv p\left(w_{a}\right)$ the adult productivity. During pure reproduction, we have $u(t)=0$ and thus the hamiltonian is

$$
H(t)=-l(t) m_{a} V(t)+p_{a} l(t)
$$

Following a stationarity principle, during reproduction the individual's reproductive value, $V(t)$, is constant since the organism no longer grows. So we have $V(t)=V_{a} \forall t \geq t_{a}$, where $V_{a} \equiv V\left(t_{a}\right)$ is the reproductive value of an adult. The canonical equation for $V(t)$ is therefore

$$
\frac{d V(t)}{d t}=-\frac{\partial H(t)}{\partial l(t)}=m_{a} V_{a}-p_{a}
$$

Since $V(t)$ is constant we must have $\frac{d V(t)}{d t}=0$ which implies

$$
V_{a}=p_{a} / m_{a}
$$

The reproductive value of an adult is therefore his productivity $\left(p_{a}\right)$ multiplied by the expectation of the time left to live $\left(m_{a}^{-1}\right)$.

We also have the canonical equation for lambda:

$$
\frac{d \lambda(t)}{d t}=-\frac{\partial H(t)}{\partial w(t)}=l(t) m_{a}^{\prime} V_{a}-p_{a}^{\prime} l(t)
$$

Where the prime denotes derivative with respect to body weight $\left(m_{a}^{\prime}=\left.\frac{d m(w)}{d w}\right|_{w=w_{a}}\right.$ and $\left.p_{a}^{\prime}=\left.\frac{d p(w)}{d w}\right|_{w=w_{a}}\right)$. Replacing $V_{a}$ by its value, $p_{a} / m_{a}$, this gives

$$
\frac{d \lambda(t)}{d t}=\left[\frac{m_{a}^{\prime} p_{a}}{m_{a}}-p_{a}^{\prime}\right] l(t)
$$

Where $l(t)=l_{a} e^{-m_{a}\left(t-t_{a}\right)}$, the probability to be still alive in $t$, is the probability $l_{a}$ of being alive at the switch age $t_{a}$, multiplied by the probability to survive after that until $t>t_{a}$.

For simplicity, we then define $s(w) \equiv m(w)^{-1}$, the life expectancy of an individual of size $w$, and thus $s_{a} \equiv m_{a}^{-1}$ the life expectancy of an individual of size $w_{a}$. Under the constraint that $\lambda(t)$ must tend toward zero at infinity, equation 6 can be integrated into

$$
\lambda(t)=\left[p_{a}^{\prime} s_{a}+s_{a}^{\prime} p_{a}\right] l_{a} e^{-m_{a}\left(t-t_{a}\right)}
$$

Hence the marginal value of body weight at the switch age is

$$
\lambda_{a} \equiv \lambda\left(t_{a}\right)=\left[p_{a}^{\prime} s_{a}+s_{a}^{\prime} p_{a}\right] l_{a}
$$

Since we know that the switching age $t_{a}$ must obey the condition $\lambda\left(t_{a}\right)=l\left(t_{a}\right)$. This gives us the necessary and sufficient condition for $t_{a}$ to be the switch age: $p_{a}^{\prime} s_{a}+s_{a}^{\prime} p_{a}=1$ (identical to equation 3 of Koziowski and Weiner, 1997 ; see also Kozłowski and Wiegert, 1987; Perrin, 1992) which writes

$$
\left(p_{a} s_{a}\right)^{\prime}=1
$$


where $p_{a} s_{a}$ is the expected total reproduction during adult life, and the prime denotes derivative with respect to body weight. The optimal switching age is thus the age at which the marginal gain of body weight in terms of expected future reproduction is exactly equal to 1 . By choosing specific production and mortality functions, the equation 7 allows us to calculate the evolutionarily stable age at maturity, $t_{a}^{*}$, and adult size, $w_{a}^{*}$, of individuals.

In the case where only productivity depends on size (constant mortality $m$ ), equation 7 simplifies to $p_{a}^{\prime}=m$. Hence, in this case, growth stops precisely when the marginal return of body size is exactly equal to mortality.

\subsubsection{Pure growth phase}

During this phase, we have $u(t)=1$, and the Hamiltonian is thus:

$$
H(t)=p[w(t)] \lambda(t)-l(t) m[w(t)] V(t)
$$

With the canonical equation for $\lambda(\cdot)$

$$
\frac{d \lambda(t)}{d t}=-\frac{\partial H(t)}{\partial w(t)}=-\lambda(t) p^{\prime}[w(t)]+l(t) V(t) m^{\prime}[w(t)]
$$

where the prime denotes derivative with respect to body weight. And the canonical equation for $V(\cdot)$ :

$$
\frac{d V(t)}{d t}=-\frac{\partial H(t)}{\partial l(t)}=m[w(t)] V(t)
$$

With the separation of variables technique, this equation gives $\frac{d V}{V}=m[w(t)] d t$, where $m(t)$ stands for $m[w(t)]$ for simplicity. Since we know the value of $V(\cdot)$ at age $t_{a}(\forall t \geq$ $\left.t_{a}, V\left(t_{a}\right)=V_{a}=p_{a} / m_{a}\right)$, this can be intergrated between any $t \leq t_{a}$ and $t_{a}$ to give:

$$
\int_{V(t)}^{V_{a}} \frac{d V}{V}=\int_{t}^{t_{a}} m(s) d s
$$

and thus $-\ln \left(\frac{V(t)}{V_{a}}\right)=\int_{t}^{t_{a}} m(s) d s$, which finally allows to derive the reproductive value during growth:

$$
V(t)=V_{a} e^{-\int_{t}^{t a} m(s) d s}
$$

Hence, as expected, the reproductive value at any age $t \leq t_{a}$ is equal to the reproductive value at adulthood, $V_{a}$, multiplied by the probability to actually reach adulthood from age $t$.

Now, recall that $l(t)=e^{-\int_{0}^{t} m(s) d s}$. Hence, the product $l(t) V(t)$ is equal to $V_{a} e^{-\int_{0}^{t a} m(s) d s}=$ $V_{a} l_{a}$. In other words, during growth, the product $l(t) V(t)$ is in fact a constant independent of $t$. In particular, this is true for $t=0$, and thus $\forall t<t_{a}, l(t) V(t)=V_{0}$, where $V_{0}$ is the reproductive value at age 0 , which is actually the same thing as the lifetime reproductive success of the individual.

Hence, finally, we have the canonical equation for $\lambda(\cdot)$ during growth:

$$
\frac{d \lambda(t)}{d t}=m^{\prime}(w) V_{0}-p^{\prime}(w) \lambda(t)
$$




\subsection{Discount rate across the life cycle}

Our aim is to derive the discount rate that individuals should express at each instant of their life. That is, we aim to answer the question: assuming individuals decision machinery has been shaped by natural selection, at what rate should they discount the future? To answer this question, we derive the marginal value of resources $v(t)$ at every instant $t$ of the life of individuals, that is the value added by an infinitesimal amount of resource obtained at time $t$, and we are interested in finding out how this marginal value changes during the life of an individual. Our reasoning is that if the decision machinery of individuals has been well calibrated by natural selection, then they should subjectively value each unit of resources in proportion to their objective marginal value with regard to fitness. As a result, individuals should express a discount rate that depends on the slope of $v(t)$ with time. At any instant $t$ of their life, if the marginal value of resources, $v(t)$, decreases sharply with time at this instant, then individuals should strongly prefer a resource now over a resource later, that is they should have a short time horizon, and conversely if $v(t)$ decreases slowly with time (or a fortiori if it is constant or increasing) then individuals should have a long time horizon.

More precisely, the evolutionarily stable discount rate $\delta(t)$ at every instant of individuals' life (that is, the discount rate they should express if their decision machinery has been well calibrated by natural selection) is the solution of the following differential equation $\dot{v}(t)=-\delta(t) v(t)$ (where the dot denotes derivative with respect to time). Hence, the evolutionarily stable exponential discount rate at time $t$ is defined as $\delta(t) \equiv-\dot{v}(t) / v(t)$.

In the present model, the marginal value $v(t)$ of a unit of resource available for the individual at time $t$ depends on the life stage the individual is in. During the growth phase (i.e. $\forall t \leq t_{a}$ ), the marginal value of a unit of resource is $\lambda(t)$ since all resources are used for growth. During the reproduction phase (i.e. $\forall t \geq t_{a}$ ), on the other hand, the marginal value of a unit of resource is $l(t)$, since all resources are used for reproduction. Hence, the fact that $\lambda(t)$ and $l(t)$ always decrease with time indicates that the marginal value of a resource is always higher when it comes early in life than late. As a result, the individuals should always discount future resources relative to present ones. The question is at what rate exactly should this discount take place?

\subsubsection{Adulthood}

We begin with the reproduction phase because it is the simplest. During this phase, the marginal value of a unit of resource is given by the survival probability $l(t)$. Since the mortality rate during adulthood is constant (equal to $m_{a}$ ), this survival probability decreases exponentially at a rate $m_{a}$. Hence the evolutionarily stable discount rate is simply $\delta(t)=m_{a}$. In this stage of life, it is therefore mortality alone that controls the time horizon of individuals. If the mortality rate is high then adults should sharply discount their future, otherwise they should discount it weakly.

\subsubsection{Growth}

During the growth phase, resources are not used to reproduce but to grow, hence the marginal value of a resource is given by $\lambda(t)$. Thus the evolutionarily stable discount rate 
is $\delta(t) \equiv-\dot{\lambda}(t) / \lambda(t)$. From equation 8 , this gives

$$
\delta(t)=p^{\prime}(w)-\frac{m^{\prime}(w)}{\lambda(t)} V_{0}
$$

where we recall that $p^{\prime}(w)>0$ and $m^{\prime}(w)<0$. Hence, the discount rate is always strictly positive.

\subsection{Risk aversion across the life cycle}

To measure individuals' risk aversion in age $t$, we imagine that the amount of resources produced by the individual at age $t$ is subject to a small perturbation $\epsilon$ (which can be positive or negative), and we calculate the eventual fitness, $V_{0}(t, \epsilon)$, of the individual who experienced that small perturbation, assuming that she behaves optimally afterwards. Following the same reasoning as in the case of time-horizon, we then assume that individuals preferences with respect to risk have been well calibrated by natural selection and we thus measure the evolutionarily stable degree of risk aversion in $t$ as

$$
\rho(t)=-\lim _{\epsilon \rightarrow 0} \frac{\partial^{2} V_{0}(t, \epsilon)}{\partial \epsilon^{2}}
$$

If $V_{0}(t, \epsilon)$ increases linearly with $\epsilon$ then individuals should be risk-neutral. If $V_{0}(t, \epsilon)$ increases concavely with $\epsilon$ then individuals should be risk-averse, since they lose more with a negative perturbation than they gain with a positive perturbation. And conversely, if $V_{0}(t, \epsilon)$ increases in a convex manner with $\epsilon$, then individuals should have an intrinsic preference for risk.

\subsubsection{Reproduction}

During adulthood, any additional resource allocated to the individual is used to reproduce. In this case, the perturbation $\epsilon$ simply changes linearly the individual's fitness by a quantity $\epsilon$. Individual should therefore be risk-neutral. That is, in their eyes, only the expected size of a resource should matter, and not its variance.

\subsubsection{Growth}

During the growth phase, we know that the perturbation $\epsilon$ does not change the optimal body weight at maturity, which is always given by equation 7 . So the only effect of $\epsilon$ is to change the age at maturity (by delaying it or advancing it slightly). By considering explicit trade-off functions (see Section 3.2), this effect on the fitness of individuals and their evolutionarily stable degree of risk aversion can be calculated numerically (see SI Section 1 and Section 2 for details).

\section{Results}

\subsection{Discounting when mortality is constant}

To gain an intuitive understanding of the dynamics of the evolutionarily stable (thereafter, ES) discount rate across individuals' life, we focus first on a simple scenario where 
body weight (that is, an individual's capital) only affects resource production, with the probability of staying alive arising from a constant exogenous mortality rate. In this case, $m^{\prime}(w)=0$, and equation 9 becomes $\delta(t)=p^{\prime}(w)$. The ES discount rate during growth is simply given by the marginal productivity of capital. In other words, an individual's ES time horizon at a given age does not depend at all on her mortality rate, but only on the marginal productivity of resources at this age. When resources can significantly improve the individual's productivity $\left(p^{\prime}(w)\right.$ is high) then she should be impatient to get them; that is, her time horizon should be short. On the contrary, when the individual's productivity is plateauing and almost no longer increases with body weight, then the individual should be patient; that is, her time horizon should be long.

If body weight has diminishing returns on productivity (that is, if $p^{\prime \prime}(w)<0$ ), the time horizon of individuals should get longer as they grow. Immature individuals, at the very beginning of their growth, have a very short time horizon, much shorter than their (constant) extrinsic mortality rate. As their capital (i.e. body weight) increases, that is as they move forward in their lives, individuals should become less and less impatient, and their ES discount rate closer and closer to their mortality rate. Individuals then switch to reproduction at the precise age where their ES discount rate becomes exactly equal to their mortality (equation 7). At this point, they stop growing and their ES discount rate remains equal to their mortality, until they die.

We remark here, and this remains true in the general case that follows, that the ES discount rate is never the sum of the effect of collection risks plus the effect of waiting costs. Rather, the ES discount rate is controlled entirely by waiting costs and completely independent of collection risks throughout the growth phase and on the contrary controlled entirely by collection risks and completely independent of waiting costs during the reproduction phase. This may seem counter-intuitive at first but is in fact a logical consequence of the evolutionarily stable bang-bang strategy expressed by individuals according to which they stop growing once they reach maturity. The ES discount rate during a given phase of an individual's life depends on what the individual has to do best with the resources she earns during that phase. During the reproduction phase, the best investment for an individual is to reproduce and never to grow. Resources are therefore never used to improve productivity and waiting costs are completely absent. On the contrary, during the growth phase, the best investment for an individual is to grow and never to reproduce. Mortality thus plays no role in the ES discount rate during that phase (for the same reason that there can be no senescence before reproduction; Baudisch, 2008). If an individual has decided not to reproduce at all anyway, then it does not make any difference that she dies possessing or not possessing any given resource. At the time of her reproductive maturity, an individual thus suddenly switches from a situation where her time horizon is entirely controlled by waiting costs to a situation where it is entirely controlled by collection risks. This transition, however, takes place precisely at the moment when the two are equal so that it does not generate any discontinuity in the ES discount rate.

\subsection{Numerical results in the general case}

In the general case where body weight affects both productivity and mortality rates, analytical derivations do not provide a clear intuitive interpretation of individuals ES 
discounting and risk profiles. Thus, in this section we provide a numerical analysis of the life cycle model, by choosing explicit trade-offs functions for productivity and mortality.

Specifically, the relationship between productivity and body weight is given by the function $p(w)=\kappa w^{\alpha}$ with $\alpha \in[0,1]$, while mortality is defined by $m(w)=\mu w^{-\beta}$, with $\beta>0$. These are usual functions in life history modelling (see e.g., Koziowski and Weiner, 1997), characterised across the range of parameters considered in our case by diminishing returns of body weight on both productivity and survival.

Here, $\alpha$ and $\beta$ measure distinct aspects of the quality of the environment. A higher value of $\alpha$ reflects a more productive environment, that is an environment in which body weight has a higher marginal effect on productivity. On the other hand, a higher value of $\beta$ reflects a safer environment, in the sense that it is enough to invest a little in body weight to significantly reduce one's mortality. In both cases, $\kappa$ and $\mu$ are used as scaling parameters.

In addition to these parameters, we also define $w_{0}$, the initial capital, which can be seen as implicitly capturing the level of parental care, considered here as an exogenous parameter, or more generally as capturing the minimum capital that any individual has at birth and could be either provided by their parents or merely present in the environment. Therefore, $w_{0}$ also captures an important aspect of the quality of the environment. Based on the specification of the mortality function, we must assume that $w_{0} \geq 1$.

With trade-offs functions fully specified, we can now compute numerically (see SI Section 1 for details) and represent graphically ES discount rates across the life cycle and for a range of scenarios (Figure 1).

Figure 1 illustrates the fact that the pattern obtained for the general case where body weight affects both mortality and productivity is consistent with the analytical results detailed in the previous section for the simpler scenario where mortality remains constant. Namely, ES discounting is still characterised by two distinct regimes: (i) during the growth phase, the ES discount rate monotonously decreases as the individuals get bigger; (ii) once the ES discount rate $\delta$ equals the mortality rate $m$, growth stops and a pure reproductive period begins during which ES discounting is exclusively controlled by mortality and remains constant until death. Although in the general case the ES discount rate $\delta$ does not correspond exactly to the marginal productivity of body weight during growth, their dynamics remain tightly linked (Figure 1).

Thus, in the case where mortality is state dependent with both diminishing returns of body weight on productivity and survival, it remains true that individuals should have a shorter time-horizon in the early stages of their life. At this time, their ES discount rate is higher than their mortality rate and captures the important benefits in terms of resource production and mortality reduction obtained by growing. As individuals get bigger, large increments in body weights are increasingly required to further obtain significant improvements in their condition, that is each unitary gain in body weight has smaller and smaller benefits, as a result waiting costs gradually vanish. Hence, individuals' time horizon becomes longer and they behave more patiently. During the phase of pure reproduction, when individuals gain a unit of resource they invest all of it into reproduction and none of it in productivity or survival. As a result, waiting costs are now absent and ES discounting depends only on collection risk, that is on mortality.

To investigate how this overall dynamic might vary across different environments, Figure 2 shows the discounting trajectories for a range of values of the parameters $\alpha$, 


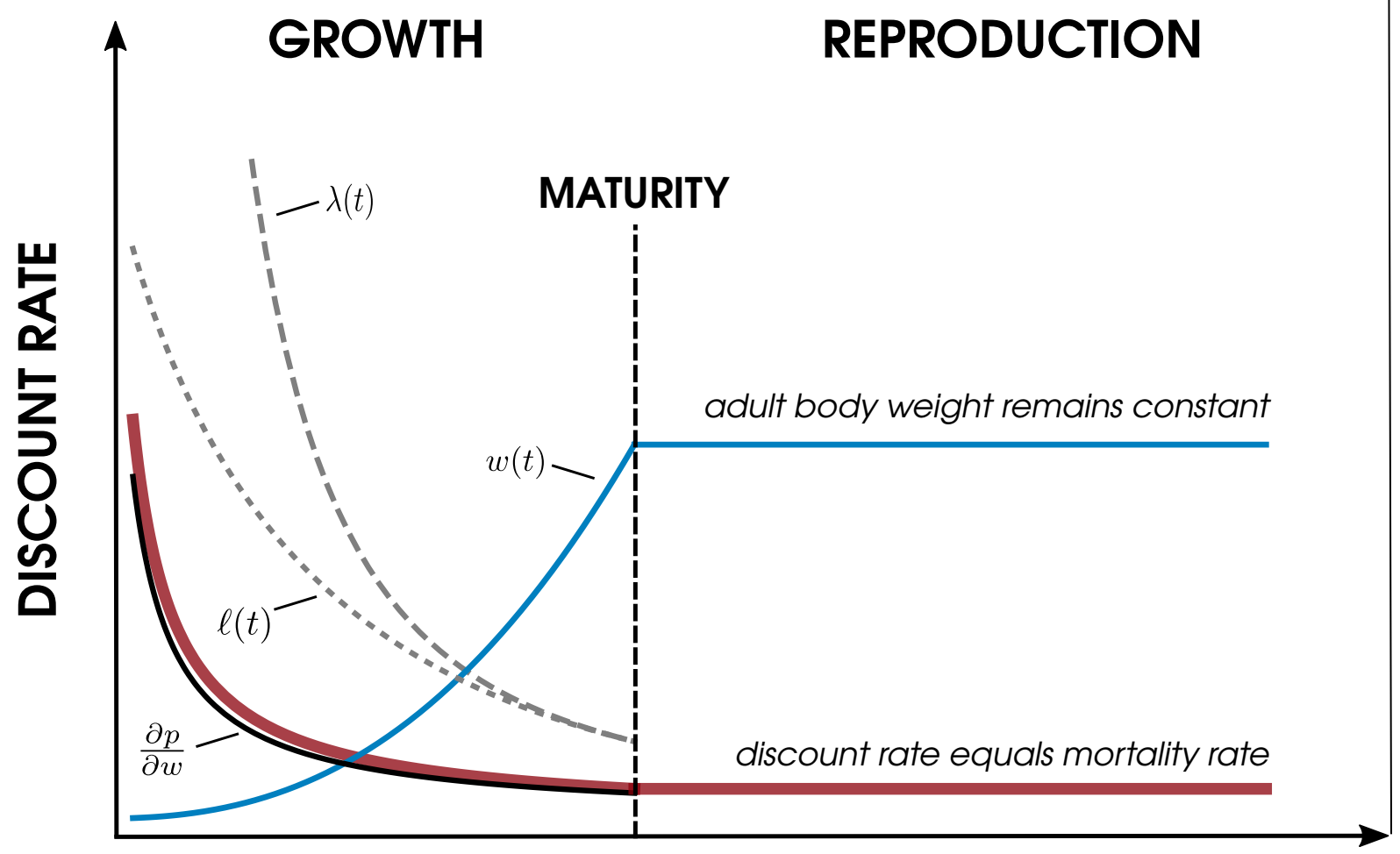

\section{TIME}

Figure 1: Time horizon across ontogeny. The diagram shows the variation in discounting throughout the optimal life cycle. During the growth phase, discounting diminishes as the marginal productivity of body size decreases. Once the marginal value $\lambda(t)$ of a unit resource equals survival $\ell(t)$, the individual matures and enters a phase of exclusive reproduction. During the reproductive stage, discount rate remains constant and is equal to the mortality rate at adult size $\mu\left(w_{\alpha}\right)$.

$\beta$ and $w_{0}$. The parameters $\alpha$ and $\beta$, which capture respectively the productivity and the safety of the environment have analogous effects on the ES discount rate $\delta$. Higher values of $\alpha$ and $\beta$ increase the ES discount rate during the early stages of growth. These trends can be explained by the fact that waiting costs directly arise from the marginal returns from investing in body weight. In an environment where little is to be gained from increasing body weight, waiting costs are therefore low.

Thus, individuals living in environments where $\alpha$ and/or $\beta$ are large have shorter ES time horizons during the initial part of the growth period compared to individuals inhabiting environments with low $\alpha$ and/or $\beta$. Yet, as the life cycle advances individuals coming from environments with higher $\alpha$ and/or $\beta$ eventually become the one discounting less. This is due to the fact that they start reproducing later in the life cycle and reach a larger body weight, resulting in a lower collection risk during the reproduction phase (Figure 2).

Regarding the third parameter $w_{0}$ however, the trend is reversed during the growth period. In this case, individuals which start with a higher initial capital $w_{0}$ are expected to discount less during their whole growth. This again comes from the impact of $w_{0}$ on the marginal returns of body weight. Since productivity and survival show diminishing returns with body weight, by definition, the larger the body weight at birth, the lower 
the marginal effect of increasing body weight. As a result, individuals born with an already large capital are spared the initial phase of high waiting costs and exhibit a longer ES time horizon throughout their growth. Because being born larger only gives a developmental headstart, however, but does not affect the body weight at which the switch to reproduction should occur (Figure 2), initially large individuals reproduce earlier but eventually everyone converges to the same ES discount rate, controlled by mortality. 

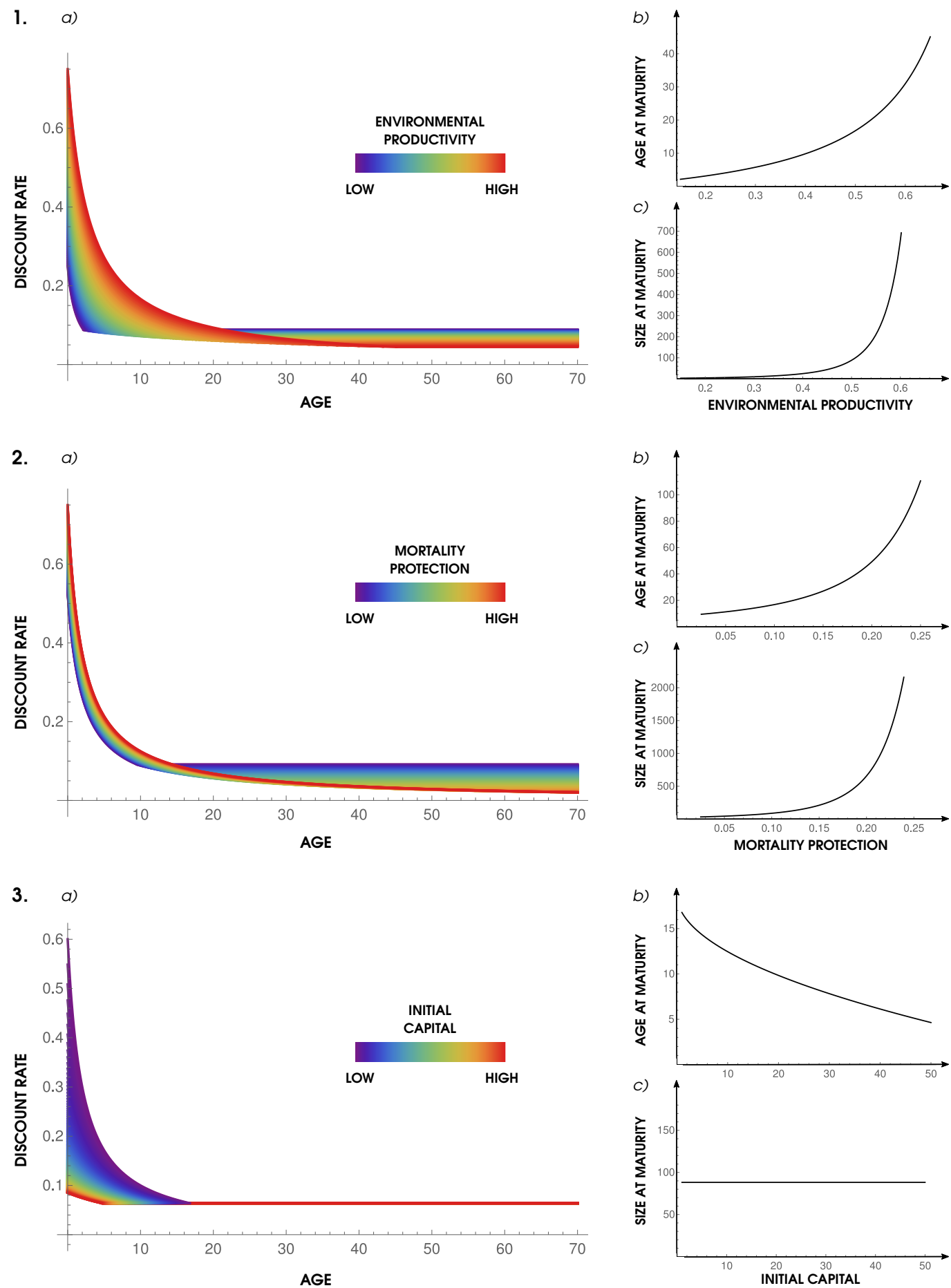

Figure 2: Time horizon across environments. 1. Variations in discount rate (a), age at maturity (b) and size at maturity (c) for increasing values of the $\alpha$ parameter of the productivity function. The optimal life cycle and discounting trajectory is computed for each increment along the gradient. A higher $\alpha$ reflects a more productive environment and is associated with greater discounting during the early phase of the growth stage (a). Yet, because a highly productive environment is associated with late maturity (b) and greater adult size (c), individuals eventually reach a lower discount rate as they keep getting bigger. 2. Variations for the same variables for increasing values of the $\beta$ parameter of the mortality function. A higher $\beta$ reflects an environment where individuals can more easily protect themselves against mortality sources. The same pattern of higher discounting during early growth (a), early switch to reproduction (b) and bigger adult size (c) is found. 3. Results obtained for increasing values of $w_{0}$, i.e. individuals' initial amount of capital. This time a high value is associated with lower discounting (a), which remains true for the whole lifecycle since maturation occurs earlier (b) and adult size is not affected by the initial capital (c). 


\subsection{Attitude toward risk}

Beyond attitude toward time, the numerical analysis of the model also provides us with information about the dynamics of attitudes towards risk across the life-cycle and for various environmental parameters (Figure 3). The overall pattern of risk sensitivity mirrors the results obtained for the discount rate. Individuals are always the most risk averse at the very beginning of their life. This aversion to risk rapidly diminishes as individuals eventually get bigger. Across environments, environments with higher values of $\alpha$ and/or $\beta$ are associated with greater risk aversion, while larger body weight at birth is associated with lower risk aversion throughout growth.

a)

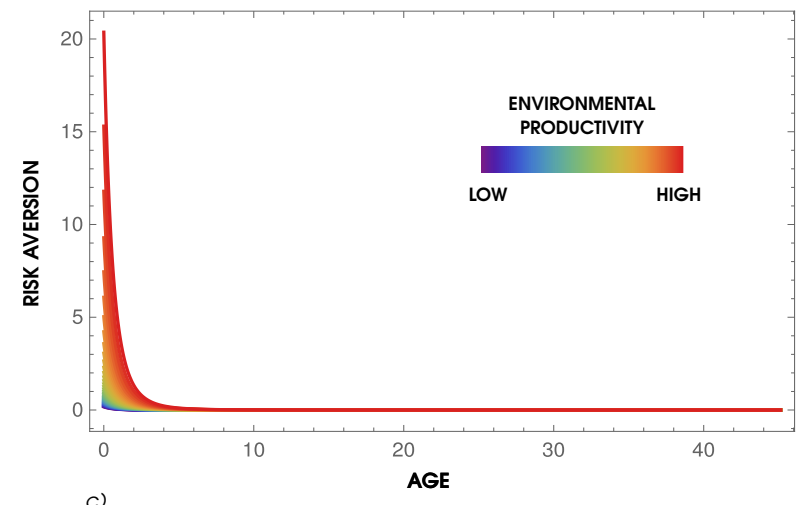

c)

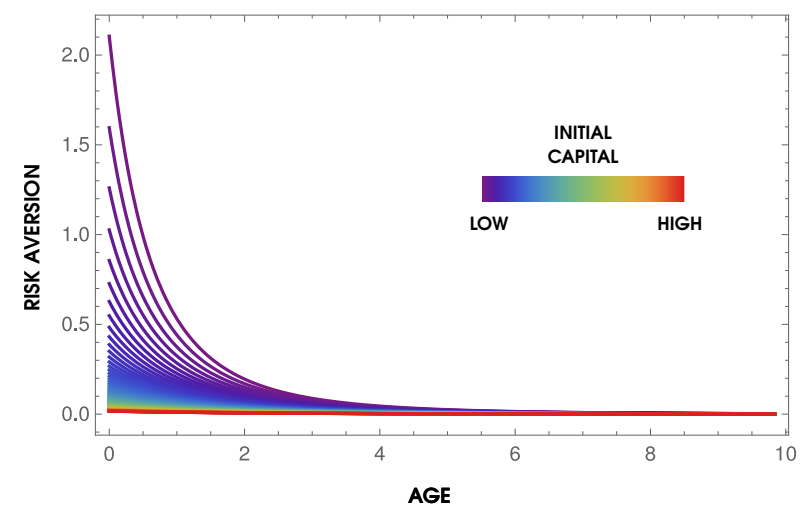

b)

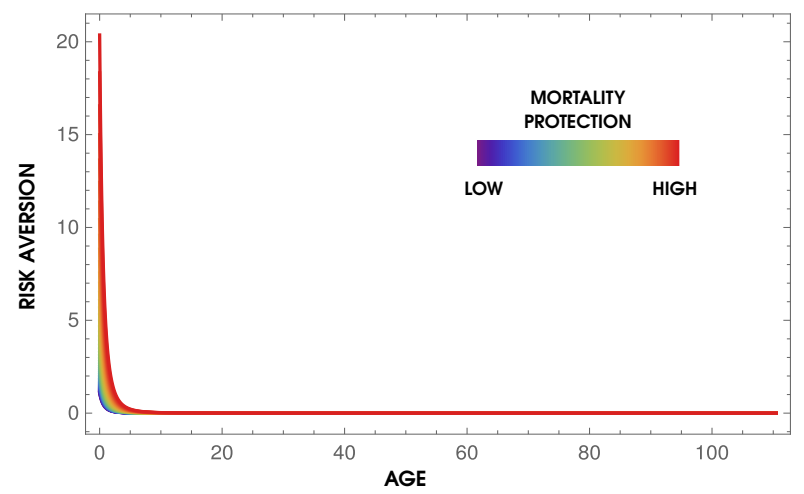

Figure 3: Risk aversion across environments. Results for risk aversion show patterns similar to those obtained for discounting, with less patient individuals being also more risk averse. While individuals are always risk neutral during the reproductive phase, a highly productive environment (increasing $\alpha$ ) is associated with more risk aversion during the growth phase (a). An environment where mortality sources are more easily neutralized is also associated with an increase in risk aversion (b). Finally, a higher level of initial capital conversely yields less risk aversion during growth. 


\section{Discussion}

In this article, we have used a simple dynamic life history model to highlight the importance of waiting costs on time-horizon across individuals' lifetime and in different environmental settings. Although collection risks (and mortality in particular) are usually invoked to explain differences in time preferences, our model shows that they are not the only, and probably not even the most important, sources of variation in temporal discounting.

When an individual must choose between obtaining a resource immediately or a larger resource later, she must take into account two elements. On the one hand, she must take into account the probability that the delayed resource will never actually be given to her, either because she will have died in the meantime or for another reason related to general environmental instability. This is the so-called collection risk. As a result of this type of risk, the more unstable the environment is, and in particular the higher the mortality rate, the more individuals should discount the future. On the other hand, beyond collection risk, when choosing between an immediate or a delayed resource an individual must also take into account all the benefits she could have earned, during the delay, by making use wisely of this resource. Obtaining a resource is not like earning points that increase the score in some kind of game. Rather, resources can be transformed into capital (Kaplan et al., 2000), that is into assets that allow the organism to better exploit its environment, either by increasing its productivity or by reducing its degradation and mortality rates, and that can be of a biological nature (the biological organism itself) or extra-biological (a shelter, a technical tool, knowledge, skills, etc.). When an individual chooses a delayed resource over an immediate one, she renounces all the benefits this supplementary capital would have generated during that delay. These forgone benefits are independent of collection risks. They are paid even if the individual is certain to obtain the resource eventually. They constitute the waiting costs. The more an individual can gain by investing new resources wisely into capital, the higher the waiting costs. Therefore, an individual who still has scope to increase significantly her productivity and/or to improve her ability to cope with environmental hazards loses much more, per unit of time waiting, than an individual who no longer has ways to improve significantly her capital and for whom gaining a resource is actually just like earning points to keep in a safe.

One can see this principle as a generalization, to individual growth, of the standard result of life history theory stating that selection favours faster strategies in growing populations than in populations at demographic equilibrium (Abrams, 1993; Caswell, 2007; Dańko et al., 2017). In a growing population, the offspring one produces are a capital that allow one's lineage to produce further offspring, and it is therefore adaptive to invest early in this capital (that is, to reproduce early). Here we show that the same effect also takes place when one's personal capital still has room for growth. An individual whose personal capital has a strong margin of progression should be more impatient to invest into this capital than an individual whose capital is plateauing.

These results allow us to shed light on the sources of variation of time-horizon. First, they allow us to better understand the variations of time horizon with age. It is adaptive for individuals to invest in different types of capital in decreasing order of priority from the most profitable down to the least profitable ones (Iwasa and Roughgarden, 1984; Maslow, 1943; Perrin, 1992). Accordingly, the return on investment of capital is likely to 
be decreasing as individuals develop. The further an individual progresses on her pyramid of needs - that is, the more capital she accumulates - and the less each additional unit of capital allows her to improve her productivity (or her resistance to hazards). This phenomenon yields individuals that should discount less as they grow, i.e. that should become more patient the bigger they get. In our simple model, individuals eventually reach an age at which they entirely stop growing and invest all their resources in reproduction, at which point their waiting cost is nil (because their capital is plateauing) and their discount rate is solely determined by collection risk (i.e. by their mortality rate). Once growth ceases it is just the probability to be alive to collect a delayed reward that matters, since resources are directly converted into as many fitness units.

Thus, owing to the existence of waiting costs, and assuming diminishing returns, discount rates should decrease as individuals accumulate more capital. This is consistent with previous findings (Chu et al., 2010) looking at the dynamics of time preferences across individuals' lifetime. Moreover, this pattern is partially supported by the empirical data available on human temporal discounting across the lifespan, which seems to support a U-shape relationship between age and discount rates (Harrison et al., 2002; Read and Read, 2004; Richter and Mata, 2018; but see also Chao et al., 2009), meaning that middle aged adults are the most patient. Nevertheless, our model only predicts the initial phase of decrease of delay discounting, not the subsequent regain of impatience as individuals enter the late stages of their life. This latter dynamic might however mirror the increase in mortality risk due to senescence (Chao et al., 2009). Since no phenomenon of senescence could emerge from our model assumptions (Baudisch, 2008), the failure of our model to capture the late increase in discounting is expected.

Second, our model also provides us with a new perspective regarding the effect of deprivation on time horizon. In this regard, we obtain two apparently contradictory results. On the one hand, we predict that individuals should have a longer time horizon if they live in an environment that can hardly be improved by investing into capital, that is in an environment where each additional unit of capital makes little difference. On the other hand, we predict that individuals should have a shorter time horizon if the basic capital they own from birth is low because, then, each unit of capital earned makes a bigger difference throughout their lives. These two opposing predictions, together with empirical observations on the actual effect of deprivation (Bruderer Enzler et al., 2014; Green et al., 1996; Harrison et al., 2002; Tanaka et al., 2010; Wang et al., 2016), lead us to suggest that, at least in most cases, deprivation does not consist in living in an environment where the effect of each unit of capital is low, but rather in possessing little at birth and having to build everything from scratch. On the contrary, well-off individuals are characterized by the fact that they possess a large amount of capital from birth, either because they receive it from their parents or because they benefit from other social investments. In wealthy social classes of modern democracies, numerous aspects of people's life are indeed facilitated by public investments such as receiving some form of health care, getting an education or using efficient means of transportation. Hence, people already come into society with a myriad of needs already taken care of, such that the influence of their own actions on their state is reduced. As an hypothesis then, we could predict that people living in societies with an important safety net and a welldeveloped welfare state should be more patient on average, compared to societies putting more emphasis on individuals taking care of their own safety. 
Moreover, the distinction between the two types of deprivation suggests some interesting additional predictions about variation in delay discounting across human societies. In poor areas where little public investment has been made and infrastructures are lacking, to the point that few opportunities are available either to improve one's productivity or to reduce one's mortality rate, comparatively low levels of discounting are expected (although high rates of mortality could compensate the absence of waiting costs). As more opportunities become available through development, however, the average discount rate in the population should rise. Interestingly, some cross-country data on time preferences shows that greater discounting is found in countries with higher economic growth rates (Wang et al., 2010). Thus, one could predict that people might exhibit especially short time horizons in highly unequal cities in the developing and developed countries where some households have very low levels of capital but opportunities to improve one's condition might still be available. More empirical data is however needed to properly assess these claims.

Interestingly, these predictions depict a somewhat different picture from accounts of individual differences in delay discounting based on collection risks. Indeed, it has been argued that people living in more deprived conditions suffer from higher collection risks because of a lack of control over future outcomes (e.g. Pepper and Nettle, 2017; RiisVestergaard and Haushofer, 2017). By contrast, in our model, discounting is actually predicted to be higher when individuals' decisions have the most impact on their state in terms of productivity and exposure to mortality.

In addition to the results obtained for delay discounting, we found identical patterns for risk aversion during ontogeny and across environments. Our model shows that the most impatient individuals should also be the most risk averse. Because capital has diminishing returns, during their growth phase, individuals face a concave "utility function". As a result, following the same logic as the standard explanation of risk aversion in expected-utility theory (Von Neumann and Morgenstern, 1944), there is always more to lose than to gain by increasing the variance in the amount of resources one earns at a given time. Individuals should therefore always be at least slightly risk-averse during their growth. But this effect decreases as individuals accumulate wealth since the benefits of further accumulation are then plateauing and the "utility function" experienced by individuals thus becomes quasi-linear. As for time preferences, the pattern predicted is mostly consistent with the empirical litterature on the relationship between income and risk aversion (e.g. Hopland et al., 2016; Tanaka et al., 2010; Wright, 2017), although the age trajectory does not seem to be supported by the available data (Dohmen et al., 2018).

Finally, despite the fact that our simple model captures well the importance of waiting costs to account for the variation in delay discounting, it suffers from important limitations that need to be acknowledged. The first limitation comes from the bang-bang nature of the optimal life history schedule arising from our formulation of the allocation problem (Baudisch, 2008; Perrin et al., 1993). Indeed, if growth is meant to include capital accumulation in a broad sense (e.g. the production of additional muscle tissue, learning a new skill, as well as the acquisition of a house), then a pure reproductive period never really occurs during the human life cycle. The initial phase of pure growth is correct but then mature individuals still invest in some form of growth and maintenance until their death. Beyond the lack of realism of a bang-bang life history strategy, this property also 
generates predictions that are at odds with part of the empirical record in the life history litterature. Indeed, the 'bang-bang' allocation schedule implies that maturity occurs as soon as a certain critical size is reached. Consequently, this actually predicts that more affluent individuals would start reproducing earlier than people starting life with only a small initial capital. Although earlier puberty with increased level of resources has been documented for girls in human population (Biro et al., 2012), the same is not true for age at first birth. Adding the possibility for individuals to invest in the quality of their children, might be a way to produce results closer to empirical data.

The last point highlights an important weakness of the model, which is that body weight at birth is completely exogenous and is not allowed to evolve in our model. Yet, as already mentioned, the amount of capital that is provided to an individual at birth by its social environment is actually a pivotal decision that might shape the allocation schedule and coevolve with time horizon. Making parental care an endogenous variable of the allocation model would therefore establish an important step forward.

In addition to more detailed aspects of the reproductive strategy like the amount of resources granted to a newborn, our model would also benefit from adding a maintenance component to the somatic strategy. Indeed, depending on the way it is implemented into a life history model, the necessity to invest resources into maintenance can alter the nature of the optimal life cycle and give rise to senescence, thus producing more realistic mortality profiles and dynamics of discounting.

Lastly, the present model is deterministic while accounting for additional sources of collection risks beyond mortality will likely require stochastic models. Introducing stochasticity would also allow the investigation of potential interactions between waiting costs and collection risks by letting the degree of stochasticity associated to a reward vary with the amount of capital. Thus, expanding the current model to take into consideration both class of factors and their interactions would constitute a significant move towards an integrated account of variation in time preferences across space and time.

\section{Supplementary Materials}

Supporting Information for the paper and the Mathematica ${ }^{\circledR}$ notebook used for the numerical analyses are publicly available at the following URL:

https://github.com/hugomell/time_is_money

\section{References}

Abrams, P. A. (1993). "Does Increased Mortality Favor the Evolution of More Rapid Senescence?" In: Evolution 47.3, pp. 877-887.

André, J.-B. and F. Rousset (2019). "Does Extrinsic Mortality Accelerate the Pace of Life? A Bare-Bones Approach". In: bioRxiv, p. 777698. DOI: 10.1101/777698.

Baudisch, A. (2008). Inevitable Aging?: Contributions to Evolutionary-Demographic Theory. Demographic Research Monographs. Berlin Heidelberg: Springer-Verlag. ISBN: 978-3-540-76655-1. DOI: 10.1007/978-3-540-76656-8. 
Biro, F. M., L. C. Greenspan, and M. P. Galvez (2012). "Puberty in Girls of the 21st Century". In: Journal of Pediatric and Adolescent Gynecology 25.5, pp. 289-294. ISSN: 10833188. DOI: $10.1016 / \mathrm{j} \cdot \mathrm{jpag} .2012 .05 .009$.

Bruderer Enzler, H., A. Diekmann, and R. Meyer (2014). "Subjective Discount Rates in the General Population and Their Predictive Power for Energy Saving Behavior". In: Energy Policy 65, pp. 524-540. ISSN: 0301-4215. DOI: 10.1016/j .enpol .2013.10. 049.

Bulley, A. and G. V. Pepper (2017). "Cross-Country Relationships between Life Expectancy, Intertemporal Choice and Age at First Birth". In: Evolution and Human Behavior. DOI: 10.1016/j.evolhumbehav.2017.05.002.

Caswell, H. (2007). "Extrinsic Mortality and the Evolution of Senescence". In: Trends in Ecology \& Evolution 22.4, pp. 173-174. ISSN: 0169-5347. DOI: 10.1016/j .tree. 2007. 01.006.

Chao, L.-W. et al. (2009). "Time Preference and Its Relationship with Age, Health, and Survival Probability". In: Judgment and Decision Making 4.1, pp. 1-19. ISSN: 19302975.

Charnov, E. L. (1993). Life history invariants: some explorations of symmetry in evolutionary ecology. Oxford: Oxford University Press. ISBN: 0198540728 (hbk.)

Chu, C. Y. C., H.-K. Chien, and R. D. Lee (2010). "The Evolutionary Theory of Time Preferences and Intergenerational Transfers". In: Journal of Economic Behavior \&5 Organization 76.3, pp. 451-464. ISSN: 0167-2681. DOI: 10.1016/j . jebo.2010.09. 011.

Dańko, M. J., O. Burger, and J. Kozłowski (2017). "Density-dependence interacts with extrinsic mortality in shaping life histories". In: PLoS ONE 12.10, pp. 1-18. ISSN: 19326203. DOI: 10.1371/journal .pone.0186661.

Dickins, T. E., S. E. Johns, and A. Chipman (2012). "Teenage Pregnancy in the United Kingdom: A Behavioral Ecological Perspective." In: Journal of Social, Evolutionary, and Cultural Psychology 6.3, pp. 344-359. ISSN: 1933-5377. DOI: 10.1037/h0099247.

Dohmen, T. et al. (2018). Identifying the Effect of Age on Willingness to Take Risks. URL: https://voxeu.org/article/effect-age-willingness-take-risks (visited on $10 / 26 / 2019)$.

Ellis, B. J. et al. (2009). "Fundamental Dimensions of Environmental Risk: The Impact of Harsh versus Unpredictable Environments on the Evolution and Development of Life History Strategies". In: Human Nature 20.2, pp. 204-268. ISSN: 1045-6767, 1936-4776. DOI: $10.1007 / \mathrm{s} 12110-009-9063-7$.

Fawcett, T. W., J. M. McNamara, and A. I. Houston (2012). "When Is It Adaptive to Be Patient? A General Framework for Evaluating Delayed Rewards". In: Behavioural Processes 89.2, pp. 128-136. ISSN: 1872-8308. DOI: 10.1016/j . beproc.2011.08.015.

Fisher, R. A. (1930). "The Genetical Theory of Natural Selection". In: Oxford: Clarendon Press, pp. 73-83.

Green, L. et al. (1996). "Temporal Discounting in Choice between Delayed Rewards: The Role of Age and Income". In: Psychology and Aging 11.1, pp. 79-84. ISSN: 0882-7974. DOI: $10.1037 / / 0882-7974.11 .1 .79$.

Hansson, I. and C. Stuart (1990). "Malthusian Selection of Preferences". In: The American Economic Review 80.3, pp. 529-544. ISSN: 0002-8282. 
Harrison, G., M. Lau, and M. B. Williams (2002). "Estimating Individual Discount Rates in Denmark: A Field Experiment". In: American Economic Review 92.5, pp. 16061617.

Hopland, A. O., E. Matsen, and B. Strøm (2016). "Income and Choice under Risk". In: Journal of Behavioral and Experimental Finance 12.C, pp. 55-64. ISSN: 2214-6350.

Houston, A. I., A. Kacelnik, and J. McNamara (1982). "Some Learning Rules for Acquiring Information". In: Functional ontogeny 1, pp. 140-191.

Iwasa, Y. and J. Roughgarden (1984). "Shoot/Root Balance of Plants: Optimal Growth of a System with Many Vegetative Organs". In: Theoretical Population Biology 25.1, pp. 78-105. ISSN: 00405809. DOI: 10.1016/0040-5809(84)90007-8.

Kageyama, J. (2009). On the Intertemporal Allocation of Consumption, Mortality and Life-History Strategies. WP-2009-008. Max Planck Institute for Demographic Research, Rostock, Germany.

Kaplan, H. et al. (2000). "A Theory of Human Life History Evolution: Diet, Intelligence, and Longevity". In: Evolutionary Anthropology: Issues, News, and Reviews 9.4, pp. 156-185. ISSN: 1520-6505. DOI: 10 . 1002/1520-6505(2000) $9: 4<156:$ : AID EVAN5>3.0. CO;2-7.

Koziowski, J. and J. Weiner (1997). "Interspecific Allometries Are By-Products of Body Size Optimization". In: The American Naturalist 149.2, pp. 352-380. ISSN: 0003-0147. DOI: $10.1086 / 285994$.

Kozłowski, J. and R. G. Wiegert (1987). "Optimal Age and Size at Maturity in Annuals and Perennials with Determinate Growth". In: Evolutionary Ecology 1.3, pp. 231-244. ISSN: 1573-8477. DOI: $10.1007 / \mathrm{BF} 02067553$.

León, J. A. (1976). "Life Histories as Adaptive Strategies". In: Journal of Theoretical Biology 60.2, pp. 301-335. ISSN: 0022-5193. DOI: 10.1016/0022-5193(76) 90062-X.

Maslow, A. H. (1943). "A Theory of Human Motivation." In: Psychological Review 50.4, pp. 370-396. ISSN: 1939-1471, 0033-295X. DOI: 10.1037/h0054346.

Mell, H., N. Baumard, and J.-B. André (2017a). "Both Collection Risk and Waiting Costs Give Rise to the Behavioral Constellation of Deprivation". In: Behavioral and Brain Sciences 40. ISSN: 0140-525X, 1469-1825. DOI: 10.1017/S0140525X17001030.

Mell, H. et al. (2017b). "Childhood Environmental Harshness Predicts Coordinated Health and Reproductive Strategies: A Cross-Sectional Study of a Nationally Representative Sample from France". In: Evolution and Human Behavior. ISSN: 1090-5138. DOI: 10.1016/j. evolhumbehav. 2017.08.006.

Nettle, D., W. E. Frankenhuis, and I. J. Rickard (2012). "The Adaptive Basis of Psychosocial Acceleration: Comment on Beyond Mental Health, Life History Strategies Articles." In: Developmental Psychology 48.3, pp. 718-721. ISSN: 1939-0599, 0012-1649. DOI: $10.1037 / \mathrm{a} 0027507$.

Pepper, G. V. and D. Nettle (2014). "Perceived Extrinsic Mortality Risk and Reported Effort in Looking after Health: Testing a Behavioral Ecological Prediction". In: Human Nature (Hawthorne, N.Y.) 25.3, pp. 378-392. ISSN: 1936-4776. DOI: 10.1007/s12110014-9204-5.

- (2017). "The Behavioural Constellation of Deprivation: Causes and Consequences". In: Behavioral and Brain Sciences 40. ISSN: 0140-525X, 1469-1825. DOI: 10.1017 / S0140525X1600234X. 
Perrin, N. (1992). "Optimal Resource Allocation and the Marginal Value of Organs". In: The American Naturalist 139.6, pp. 1344-1369. ISSN: 0003-0147, 1537-5323. DOI: $10.1086 / 285390$.

Perrin, N., R. M. Sibly, and N. K. Nichols (1993). "Optimal growth strategies when mortality and production rates are size-dependent". In: Evol. Ecol. 7.1982, pp. 576592.

Perrin, N. and R. M. Sibly (1993). "Dynamic Models of Energy Allocation and Investment". In: Annual Review of Ecology and Systematics 24.1, pp. 379-410. ISSN: 00664162. DOI: 10.1146/annurev.es.24.110193.002115.

Pontriagin, L. S., L. W. Neustadt, and L. S. Pontriagin (1986). The Mathematical Theory of Optimal Processes. English ed. Classics of Soviet Mathematics v. 1. New York: Gordon and Breach Science Publishers. 360 pp. ISBN: 978-2-88124-077-5.

Read, D. and N. Read (2004). "Time Discounting over the Lifespan". In: Organizational Behavior and Human Decision Processes 94.1, pp. 22-32. ISSN: 07495978. DOI: 10. 1016/j.obhdp. 2004.01 .002$.

Richter, D. and R. Mata (2018). "Age Differences in Intertemporal Choice: U-Shaped Associations in a Probability Sample of German Households." In: Psychology and Aging 33.5, pp. 782-788. ISSN: 1939-1498, 0882-7974. DOI: 10.1037/pag0000266.

Riis-Vestergaard, M. I. and J. Haushofer (2017). "Stuff Goes Wrong, so Act Now". In: Behavioral and Brain Sciences 40. ISSN: 0140-525X, 1469-1825. DOI: 10.1017/S0140525X17001091.

Rogers, A. R. (1994). "Evolution of Time Preference by Natural Selection". In: The American Economic Review 84.3, pp. 460-481. ISSN: 0002-8282.

Shaw, M., H. Tunstall, and D. Dorling (2005). "Increasing Inequalities in Risk of Murder in Britain: Trends in the Demographic and Spatial Distribution of Murder, 1981-2000." In: Health \& place 11.1, pp. 45-54. DOI: 10.1016/j.healthplace.2004.01.003.

Sozou, P. D. and R. M. Seymour (2003). "Augmented Discounting: Interaction between Ageing and Time-Preference Behaviour". In: Proceedings. Biological Sciences 270.1519, pp. 1047-1053. ISSN: 0962-8452. DOI: 10.1098/rspb.2003.2344.

Stevens, J. R. and D. W. Stephens (2010). "The Adaptive Nature of Impulsivity". In: Impulsivity: The Behavioral and Neurological Science of Discounting. Washington, DC, US: American Psychological Association, pp. 361-387. ISBN: 978-1-4338-0477-9 978-1-4338-0820-3. DOI: 10.1037/12069-013.

Tanaka, T., C. F. Camerer, and Q. Nguyen (2010). "Risk and Time Preferences: Linking Experimental and Household Survey Data from Vietnam". In: American Economic Review 100.1, pp. 557-571. ISSN: 0002-8282. DOI: 10.1257/aer.100.1.557.

Taylor, H. M. et al. (1974). "Natural Selection of Life History Attributes: An Analytical Approach". In: Theoretical Population Biology 5.1, pp. 104-122. ISSN: 00405809. DOI: 10.1016/0040-5809(74)90053-7.

Von Neumann, J. and O. Morgenstern (1944). Theory of Games and Economic Behavior. Princeton: Princeton University Press.

Wang, M., M. O. Rieger, and T. Hens (2010). "How Time Preferences Differ: Evidence from 45 Countries". In: SSRN Electronic Journal. ISSN: 1556-5068. DOI: 10 . 2139 / ssrn. 1481443.

- (2016). "How Time Preferences Differ: Evidence from 53 Countries". In: Journal of Economic Psychology 52, pp. 115-135. ISSN: 01674870. DOI: 10.1016/j.joep. 2015. 12.001. 
Winding, T. N. and J. H. Andersen (2015). "Socioeconomic Differences in School Dropout among Young Adults: The Role of Social Relations". In: BMC Public Health 15. ISSN: 1471-2458. DOI: 10.1186/s12889-015-2391-0.

Wright, J. (2017). To What Extent Does Income Predict an Individual's Risk Profile in the UK (2012- 2014). 80757. University Library of Munich, Germany. 